\title{
Reply to Letter to the Editor: Re: Preoperative versus intraoperative endoscopic sphincterotomy for management of common bile duct stones. Surg Endosc (Online first)
}

\author{
Ahmed Abdel-Raouf El-Geidie
}

Published online: 27 February 2011

(C) Springer Science+Business Media, LLC 2011

First, I thank Professor Nair for his interest in our article. On behalf of all the authors, I reply to his valuable comments.

The method of randomization followed in our study was a sealed envelopes method. This method is described in the Patients and Methods section as follows: "Patients fulfilling the criteria of enrollment underwent MRCP, and only patients with MRCP evidence of CBD stones were eligible for randomization. After obtaining consent, eligible patients were randomized into two groups using sealed opaque envelopes." The envelopes were opened at the outpatient clinic by a secretary not involved in the trial.

In this trial, patients had a single intravenous dose of third-generation cephalosporin $1 \mathrm{~h}$ before endoscopic retrograde cholangiopancreatography (ERCP) and laparoscopic cholecystectomy (LC). No antibiotics were administered intra- or postoperatively except in cases of acute cholecystitis, for which two doses of a third-generation cephalosporin were given postoperatively. Wound infection at a port site (epigastric port) after LC was reported and managed by frequent dressings with oral antibiotics.

I agree that common bowel duct (CBD) stenting makes subsequent surgery more demanding. Only one of the three patients who had failure of stone extraction in the preoperative endoscopic sphincterotomy/laparoscopic cholecystectomy group experienced difficulty at open surgery. In this case, the operating time was $120 \mathrm{~min}$.

Also, as mentioned in the trial, bile leak in the surgical drain was minor ( $\sim 50 \mathrm{ml} /$ day) and stopped without any intervention after 3 days. Finally, I hope I have clarified the comments proposed by Professor Nair.
A. A.-R. El-Geidie ( $\square)$

Gastroenterology Surgical Center, Mansoura, Egypt

e-mail: ahmedraoaf@mans.edu.eg 